\title{
ReSPUESTAS FISIOLÓGICAS A LA SEQUÍA, DE CINCO ESPECIES DE ORQUÍDEAS EPÍFITAS, EN DOS SELVAS SECAS DE LA PENÍNSULA DE YUCATÁN
}

\author{
Edilia de la Rosa-Manzano'; José Luis Andrade ${ }^{1,2,4}$; Gerhard Zotz ${ }^{3}$ \\ y CaSANDRA Reyes-García ${ }^{1}$ \\ ${ }^{1}$ Unidad de Recursos Naturales, Centro de Investigación Científica de Yucatán, A. C., Mérida, Yucatán, México \\ ${ }^{2}$ Unidad de Ciencias del Agua, Centro de Investigación Científica de Yucatán, A. C., Cancún, Quintana Roo, México \\ ${ }^{3}$ Ecología Funcional, Instituto de Biología y Ciencias ambientales, Universidad de Oldenburg, Oldenburg, Alemania \\ ${ }^{4}$ Autor para la correspondencia: andrade@cicy.mx
}

\begin{abstract}
Resumen: El dosel de las selvas secas presenta cambios anuales drásticos de luz y disponibilidad de agua, debido a que la mayoría de los árboles pierde sus hojas durante la temporada de sequía. Las epífitas que ahí habitan presentan una morfología y fisiología adecuadas para tolerar la escasez de agua y el exceso de luz. Se estudiaron las respuestas fisiológicas de cinco especies de orquídeas epífitas en relación con la variación vertical del microambiente y a la estacionalidad en una selva baja caducifolia y en una selva mediana subcaducifolia, durante las temporadas de lluvias, nortes y sequía. Los cambios fisiológicos en las orquídeas fueron debido al efecto de los cambios estacionales en ambas selvas. Las orquídeas mostraron diferentes estrategias de tolerancia a la sequía: las hojas de Encyclia nematocaulon, Cohniella yucatanensis y Laelia rubescens, de la selva baja, mantuvieron su estado hídrico constante durante el año, pero disminuyeron su tasas de fotosíntesis durante la sequía. Aunque el estado hídrico foliar de $E$. nematocaulon y Lophiaris oerstedii, de la selva mediana, disminuyó durante las sequía, la fotosíntesis no disminuyó en L. oerstedii. Cohniella ascendens no mostró variación fisiológica estacional en sus hojas; se sugiere que esto fue debido a su baja densidad estomática y a la inclinación de las mismas, porque ello evita la perdida de agua y la exposición directa a la radiación. La especie más abundante, E. nematocaulon, mostró reducción foliar y estomas pequeños, lo que le confirió gran plasticidad morfológica y fisiológica para tolerar la alta radiación y la sequía en ambas selvas.
\end{abstract}

Palabras clave: contenido relativo de agua, fotoinhibición, metabolismo ácido de las crasuláceas, potencial osmótico, selvas secas.

\begin{abstract}
Dry forest canopies exhibit drastic changes of light and water availability throughout the year, because most trees drop their leaves during the dry season. Epiphytes that inhabit there show a suit of morphological and physiological traits to tolerate water scarcity and light excess. We studied the physiological responses of five epiphyte orchid species in relation to their vertical microenvironment and the seasonality in a deciduous tropical forest and a semi-deciduous tropical forest, during the wet, early dry and dry seasons. Physiological variation of orchids was mainly seasonal in both forests. Orchids showed different strategies to cope with drought: Encyclia nematocaulon, Cohniella yucatanensis and Laelia rubescens from the deciduous forest maintained their leaf water status constant throughout the year, with reduced photosynthetic rates during the dry season. Although the leaf water status of both, E. nematocaulon and Lophiaris oerstedii from the semi-deciduous forest, diminished during the dry season, photosynthesis did not decline in L. oerstedii. Leaves of Cohniella ascendens did not show significant seasonal physiological variation, arguably due to low stomatal density and tilted leaf orientation, which reduced water loss and exposure to high radiation. The most abundant species, E. nematocaulon, showed small stomata, reduced leaf area and morphological and physiological plasticity to tolerate high radiation and drought in both forests.
\end{abstract}

Key words: crassulacean acid metabolism, dry forests, osmotic potential, photoinhibition, relative water content.

$\mathbf{L}$ as epífitas son un elemento conspicuo de los bosques tropicales, pero su diversidad y abundancia disminuye drásticamente en las selvas secas (Gentry y Dodson, 1987). Por la marcada estacionalidad, las epífitas que crecen en las selvas secas pueden recibir hasta nueve veces más luz du- rante la temporada de sequía, en comparación con la temporada de lluvias (Graham y Andrade, 2004; Andrade et al., 2006). Si bien este gradiente vertical lumínico en las selvas secas no es tan grande como en las selvas lluviosas, sí influye en la estratificación vertical en función de la resistencia a 
la sequía de algunas especies de epífitas (Graham y Andrade, 2004; Reyes-García et al., 2012).

Las epifitas presentan una morfología y fisiología modificada para tolerar la escasez de agua en los ambientes del dosel, la cual es el principal factor limitante para el crecimiento y supervivencia de estas plantas (Benzing, 1990; Zotz y Hietz, 2001; Andrade, 2003). Algunas modificaciones son la presencia de tricomas o escamas, estomas hundidos, órganos especializados para almacenar el agua (pseudobulbos en orquídeas y hojas arregladas como tanque en las bromeliáceas) y la asimilación de $\mathrm{CO}_{2}$ por medio del metabolismo ácido de las crasuláceas (CAM, por sus siglas en inglés; Winter, 1985; Benzing, 1990). La fotosíntesis CAM permite conservar el agua porque la transpiración se reduce al abrirse los estomas casi exclusivamente durante la noche, cuando el gradiente de presión de vapor entre el aire y la planta es mucho más bajo que durante el día (Nobel, 2009; Andrade et al., 2007).

Las epífitas CAM tienden a ocupar las zonas expuestas y altas del dosel, donde experimentan una combinación de sequía, luz y temperatura mayores, lo que suele causar fotoinhibición en diversas especies (Griffiths y Smith, 1983; Powles, 1984; Demmig-Adams y Adams, 1992; Graham y Andrade, 2004; Reyes-García et al., 2012). La fotoinhibición ocurre por una sobre-excitación de los centros de reacción del fotosistema II, lo que disminuye la eficiencia del aparato fotosintético y por consiguiente, el crecimiento vegetal (Björkman y Demmig, 1987).

En las selvas secas, las orquídeas y las bromeliáceas son los tipos principales de epífitas, pero existen allí con una menor diversidad y abundancia que en las selvas más húmedas (Gentry y Dodson, 1987). Sin embargo, como las selvas secas son uno de los ecosistemas tropicales más amenazados por diferentes actividades como la deforestación, la agricultura y la invasión humana (Trejo y Dirzo, 2000), las epífitas de estas selvas son un grupo vulnerable. Por tanto, se considera necesario estudiar las respuestas fisiológicas y morfológicas al ambiente, de las orquídeas epífitas que crecen en dos selvas secas de la península de Yucatán con diferente precipitación anual, para entender el funcionamiento de estas plantas en estos ecosistemas.

En estas dos selvas, se estudiaron cinco especies de orquídeas epífitas y se planteó la hipótesis de que las orquídeas que se encuentran a mayor altura en el hospedero, experimentan una disminución en su estado hídrico y fotosintético en comparación con las que crecen a menor altura dentro del dosel del hospedero. Asimismo, se espera que en la temporada de sequía disminuyan estas variables fisiológicas en comparación con las temporadas de nortes y lluvias.

\section{Materiales y métodos}

Sitios de estudio. El trabajo se realizó en dos selvas secas de la península de Yucatán, México: (1) la selva baja ca- ducifolia de Celestún (20 50'53” N, 90 14'17” O), con una precipitación media anual de $770 \mathrm{~mm}$ y una temperatura media anual de $27^{\circ} \mathrm{C}$. Los árboles alcanzan entre los 8 y 10 $\mathrm{m}$ de altura, y la mayoría de estos pierde sus hojas en la temporada de sequía (marzo a mayo). (2) La selva mediana subcaducifolia de la Reserva Biocultural Kaxil-Kiuic (20 06' $33^{\prime \prime} \mathrm{N}, 89^{\circ} 32^{\prime} 55^{\prime}$ ' O), con una precipitación media anual de $1,200 \mathrm{~mm}$ y una temperatura media anual de $26^{\circ} \mathrm{C}$. La altura de los árboles oscila entre los 13 y $18 \mathrm{~m}$, y de 50 a $75 \%$ de ellos pierden sus hojas durante la temporada de sequía (Orellana, 1999, Dupuy et al., 2012). En ambas selvas, la temporada de sequía es seguida por la temporada de lluvias (junio-octubre) y un periodo llamado localmente "nortes", el cual se caracteriza por vientos fuertes (> $80 \mathrm{~km} / \mathrm{h}$ ), lluvias escasas (20-60 $\mathrm{mm}$ en total) y temperatura diaria promedio por abajo de $20^{\circ} \mathrm{C}$ (Orellana, 1999).

Especies de estudio. Se estudiaron cinco especies de orquídeas con diferente forma de hoja: cilíndrica, ovalada y lineal. En las selva baja caducifolia: Encyclia nematocaulon (BC Rich.) Acuña, Cohniella yucatanensis Cetzal y Carnevali y Laelia rubescens Lindley; y en la selva mediana subcaducifolia: $E$. nematocaulon, Cohniella ascendens (Lindley) Christenson y Lophiaris oerstedii (Rchb. F.) A.Jimenez, Carnevali y Dressler. Las orquídeas $C$. ascendens, L. rubescens y L. oerstedii se distribuyen en México y América Central, E. nematocaulon se distribuye en México y el Caribe, mientras que C. yucatanensis es endémica de la Península de Yucatán (Carnevali et al., 2001; Cetzal-Ix y Carnevali, 2010).

Microambiente. El hospedero más frecuente para las orquídeas epífitas en ambas selvas fue Gymnopodium floribundum Rolfe (Poligonaceae), por tanto, en esta especie se realizaron las mediciones microambientales en dos estratos del principal hospedero $(3.5$ y $1.5 \mathrm{~m}$; donde las orquídeas fueron más abundantes), y por encima del dosel. El flujo fotónico fotosintético (FFF) se midió usando fotodiodos (Hamamatsu, Bridgewater, Nueva Jersey, EUA), previamente calibrados con un sensor cuántico (LI190S, LI-COR, Lincoln, Nevada, EUA); la temperatura y la humedad relativa del aire se midieron con sensores Vaisala (HMP35C-L, Campbell Scientific, Inc., Logan, Utah, EUA); la temperatura de la hoja se midió con termopares adheridos a la superficie adaxial, solamente en las temporadas de nortes y sequía (cuando las orquídeas reciben mayor luz); el déficit de presión de vapor se calculó de acuerdo a Jones (1992), con base en la temperatura foliar y la del aire. Todos los sensores se conectaron a un registrador de datos (CR21X and CR10X, Campbell Scientific, Logan, Utah, EUA), y el promedio de cada 10 min se registró en un periodo de 6 a 10 días en cada selva durante las tres temporadas.

Variables fisiológicas.

Contenido relativo de agua (CRA).- Se recolectaron mues- 
tras de hojas de plantas (aproximadamente $5 \mathrm{~cm}^{2}$ de hojas totalmente expandidas) a 3.5 y $1.5 \mathrm{~m}$ de altura en el hospedero $(n=5)$ durante las temporadas de lluvias, nortes y sequía. Las muestras se colocaron en una bolsa de plástico con un papel húmedo, se transportaron en un recipiente con hielo al laboratorio. Se obtuvo el peso fresco de las muestras y se colocaron en agua destilada por $24 \mathrm{~h}$ para obtener el peso turgente. Finalmente las muestras se secaron en una estufa a $65^{\circ} \mathrm{C}$ por $24 \mathrm{~h}$. El CRA se calculó mediante: [(peso fresco - peso seco) / (peso turgente - peso seco) $\times 100$.

Potencial osmótico.- Se recolectaron segmentos de hojas de plantas (aproximadamente $5 \mathrm{~cm}^{2}$ ), en dos alturas del hospedero ( 3.5 y $1.5 \mathrm{~m}$ ), en pre-alba, durante las tres temporadas del año $(n=5)$. Las muestras se almacenaron en nitrógeno líquido hasta su análisis en el laboratorio, donde fueron maceradas en un mortero, el líquido tisular fue absorbido con un papel filtro y el potencial osmótico fue obtenido usando un osmómetro (VAPRO 5520, Wescor, Logan, Utah, EUA).

Acidez tisular $\left(\Delta \mathrm{H}^{+}\right)$.- En las tres temporadas del año se recolectaron muestras de hojas de plantas $\left(1 \mathrm{~cm}^{2}\right.$ para Laelia rubescens y Lophiaris oerstedii; $3 \mathrm{~cm}^{2}$ para el resto de las especies; $\mathrm{n}=5$ ), en dos alturas en el hospedero ( 3.5 y $1.5 \mathrm{~m}$ ), al atardecer y a pre-alba del día siguiente, y se almacenaron en etanol $(60 \% \mathrm{v} / \mathrm{v})$. La titulación se realizó usando un titulador automático (SM Titrino 702, Herisau, Suiza) siguiendo

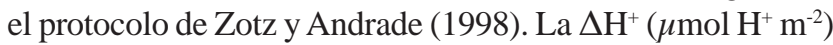
fue estimada como: $\mathrm{H}^{+}{ }_{\text {(pre-alba) }}-\mathrm{H}^{+}{ }_{\text {(atardecer) }}$.

Parámetros de la fluorescencia de la clorofila.- Se midieron usando un medidor de fluorescencia de la clorofila de amplitud modulada (Mini-PAM, Heinz Walz, Alemania), en las tres temporadas del año. La eficiencia cuántica máxima $\left(\mathrm{F}_{\mathrm{v}} / \mathrm{F}_{\mathrm{m}}\right.$; proporción de la fluorescencia variable y máxima), se midió en pre-alba (6:00 h), en plantas en dos alturas del hospedero $(3.5$ y $1.5 \mathrm{~m})$; para detalles de la nomenclatura ver Maxwell y Johnson (2000). Las curvas de respuesta a la luz se realizaron para determinar la tasa de transporte de electrones máxima (ETR ${ }_{\text {máx }}$; Rascher et al., 2000). Una hoja de cada especie fue cubierta con una bolsa de tela negra para simular condiciones de oscuridad por $30 \mathrm{~min}$. Las curvas de luz fueron realizadas a las 9:00 $\mathrm{h}$, y se aplicaron nueve pulsos de luz actínica (en el intervalo de 0-900 $\mu \mathrm{mol} \mathrm{m}^{-2}$ $\left.\mathrm{s}^{-1}\right)$ de $10 \mathrm{~s}$ de duración cada uno $(\mathrm{n}=3$ hojas de diferentes individuos).

\section{Variables morfológicas.}

Volumen del pseudobulbo.- Se seleccionaron al azar diez individuos de cada especie en cada sitio de estudio, independientemente de la altura en el hospedero, y se midieron el diámetro y la longitud del pseudobulbo. Se calculó el volumen del pseudobulbo, considerándolo como un cuerpo cilíndrico para Cohniella yucatanensis, C. ascendens y Lophiaris oerstedii y un cuerpo cónico para Encyclia nematocaulon y Laelia rubescens.
Densidad estomática.- Se recolectaron al azar cuatro hojas (de la parte media de la planta y totalmente expandidas), de cada especie, independientemente de la altura en el hospedero y por cada sitio de estudio. Se tomaron impresiones de ambas caras de la epidermis de la hoja usando un esmalte transparente para uñas, la impresión se montó en un portaobjetos. Se contaron los estomas en diez campos diferentes y se calculó la media; la densidad de estomas se calculó como: número de estomas / área observada. Las observaciones se hicieron en un microscopio óptico (Leica EDM) utilizando el aumento 10X.

Área foliar específica.- Se calculó como el área foliar dividida por el peso foliar seco. Se recolectaron al azar las hojas de orquídeas ( $n=21-34$ por especie), independientemente de la altura en el hospedero, en ambas selvas, y se midió el área foliar usando un medidor de área foliar. Para Cohniella ascendens se consideró el área de un cilindro. El peso seco se realizó de la misma forma que para el CRA.

Microscopía electrónica de barrido.- Se recolectaron muestras de hojas de individuos (de la parte media de la planta y aproximadamente $1 \mathrm{~cm}^{2}$ ), aleatoria e independientemente de la altura en el hospedero. Se hicieron cortes de aproximadamente $5 \times 5 \mathrm{~mm}^{2}$ y las observaciones se hicieron en un microscopio electrónico de barrido (MEB; Jeol, JSM-6360LV). Se midió el largo y ancho de los estomas y se calculó el área. Las muestras fueron deshidratadas utilizando diferentes concentraciones de etanol, posteriormente se secaron a un punto crítico con $\mathrm{CO}_{2}$ (SAMDRI 795, semiautomática). Finalmente se realizó la fijación de las muestras en un portaobjetos metálico de cobre y se cubrieron con una capa de oro-paladio utilizando una metalizadora (Denton Vacuum Desk II a $15 \mathrm{kV}$ ). Para mayor detalle de la preparación de las muestras ver GonzálezSalvatierra et al. (2013).

Análisis estadísticos. Para comparar las variables fisiológicas (CRA y $\Delta \mathrm{H}^{+}$) de cada especie, se usó un análisis de varianza anidado, se consideraron los estratos en el hospedero anidados en el factor árbol en cada sitio de estudio; cuando los datos no fueron normales se aplicó la prueba de KruskalWallis. Se aplicó un análisis de varianza de dos vías para evaluar las posibles diferencias del FFF a nivel de estratos y temporadas en cada selva. Cuando la prueba paramétrica fue significativa, se compararon las medias mediante la prueba de Tukey. Para comparar las variables fisiológicas (CRA y $\Delta \mathrm{H}^{+}$) entre estratos en el hospedero, se aplicó una prueba $t$ de Student pareada y, para comparar las mismas variables entre selvas se aplicó una prueba $t$ de Student. Para evaluar la densidad estomática entre especies de cada selva se aplicó un análisis de varianza de una vía y, para comparar la densidad estomática entre selvas se usó una prueba $t$ de Student. Para comparar el área foliar específica entre las especies de cada selva se aplicó una prueba de Kruskall-Wallis. Valores de $P=0.05$ fueron aceptados como significativos. Las prue- 
EdILIA DE LA RosA-Manzano ET AL.

bas estadísticas fueron realizadas con el paquete STATISTICA v.10 (Statsoft Inc., EUA).

\section{Resultados}

Luz, temperatura de la hoja y déficit de presión de vapor. Los cambios estacionales del flujo fotónico fotosintético (FFF), en el dosel, fueron sustanciales en ambas selvas (ANOVA de dos vías $F_{2,18}=310, P=0.05$ para ambas selvas; Figura 1A-C, G-I). En la selva baja caducifolia, el FFF diario promedio fue dos veces mayor durante la sequía en comparación con las otras temporadas. Las orquídeas recibieron en promedio aproximadamente tres veces más FFF a $3.5 \mathrm{~m}$ de altura $\left(32 \mathrm{~mol} \mathrm{~m}^{-2} \mathrm{~d}^{-1}\right)$ y seis veces más a $1.5 \mathrm{~m}(12$ mol m $\mathrm{m}^{-2} \mathrm{~d}^{-1}$ durante la sequía, comparada con la temporada de lluvias (11 y $2 \mathrm{~mol} \mathrm{~m}^{-2} \mathrm{~d}^{-1}$ a 3.5 y $1.5 \mathrm{~m}$ de altura, respectivamente; Figura $1 ; P=0.05$ ).

En la selva mediana subcaducifolia, los cambios en el FFF que recibieron las orquídeas durante el año fueron más pronunciados que en la selva baja, pero los valores absolutos fueron consistentemente bajos. Durante la temporada de lluvias, las orquídeas recibieron 2 y $0.7 \mathrm{~mol} \mathrm{~m}^{-2} \mathrm{~d}^{-1}$ a $3.5 \mathrm{y}$ $1.5 \mathrm{~m}$ de altura, respectivamente (Figura1G). El FFF diario promedio fue notablemente mayor durante la sequía, lo cual significa que las hojas recibieron 10 y $8 \mathrm{~mol} \mathrm{~m}^{-2} \mathrm{~d}^{-1}$ a $3.5 \mathrm{y}$

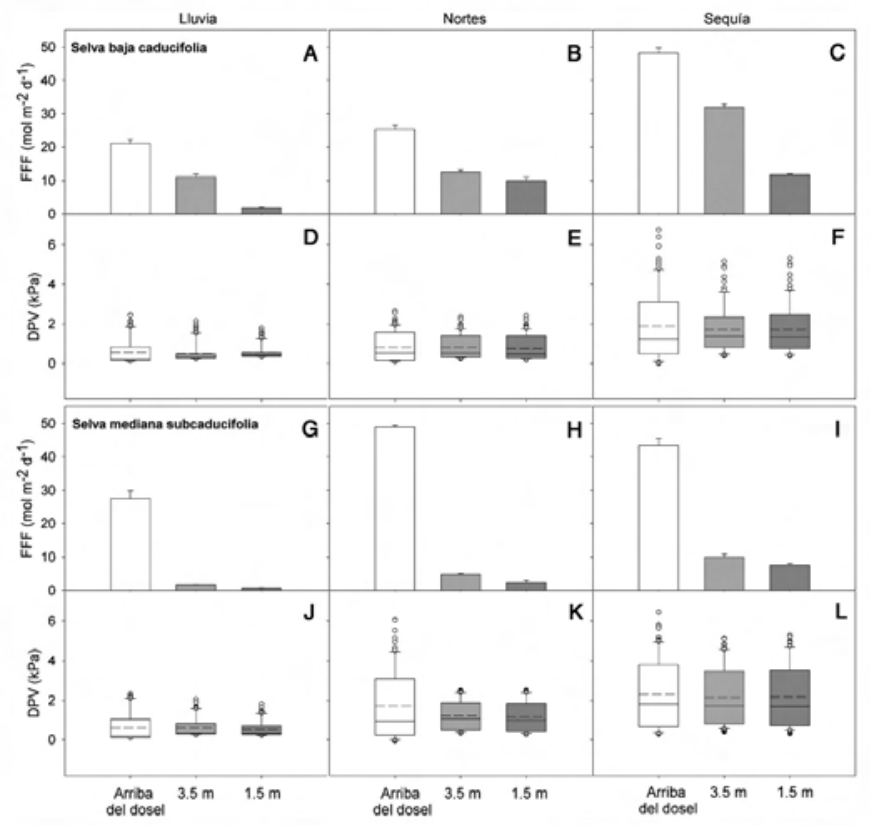

Figura 1. Flujo fotónico fotosintético (FFF; mol m $\mathrm{m}^{-2} \mathrm{~d}^{-1}$ ), déficit de presión de vapor (DPV; $\mathrm{kPa}$ ), ambos medidos arriba del dosel y en dos alturas en el árbol (3.5 y $1.5 \mathrm{~m}$ ), durante lluvias, nortes y sequía en la selva baja caducifolia de Celestún y en la selva mediana subcaducifolia de Kaxil-Kiuic. Los datos del FFF son medias \pm EE. El diagrama de caja muestra el valor mínimo, máximo, media (línea punteada) y mediana (línea continua) del DPV, n =7-10 días para ambas variables.
$1.5 \mathrm{~m}$ de altura, respectivamente, sin diferencias significativas entre estratos $(t=2.30$, g.l. $=6, P=0.05$; Figura 1I).

Paralelo al incremento del FFF, las orquídeas experimentaron un incremento de temperatura foliar durante la sequía. Por ejemplo, en la selva baja, la temperatura mínima y máxima de las hojas aumentó $\sim 2$ y $10^{\circ} \mathrm{C}$, comparadas con la temporada de nortes (Cuadro 1). Mientras que en la selva mediana el aumento de la temperatura foliar mínima y máxima fue de $\sim 2$ y $6{ }^{\circ} \mathrm{C}$, respectivamente, comparadas con la temporada de nortes (Cuadro 1).

El déficit de presión de vapor del ambiente (DPV) mostró poca variación entre los estratos en el hospedero (3.5 y $1.5 \mathrm{~m}$ ) durante las tres temporadas, por lo que se considera una media por temporada en este parámetro para las siguientes comparaciones. En la selva baja, durante la sequía, el DPV mínimo $(0.48 \pm 0.07 \mathrm{kPa})$ se duplicó en comparación con la temporada de nortes $(0.23 \pm 0.1 \mathrm{kPa})$, y el DPV máximo se incrementó de $1.9 \pm 0.2$, en nortes, a $4.6 \pm$ $0.4 \mathrm{kPa}$, en la sequía (Figura 1E, F). En la selva mediana, el DPV mínimo aumentó un 50\% en la temporada de sequía en comparación con la temporada de nortes (Figura 1K, L). Asimismo, el DPV máximo fue $80 \%$ mayor en la sequía comparada con la temporada de nortes (Figura 1K, L).

Cambios fisiológicos estacionales. Sorprendentemente, el contenido relativo de agua (CRA) de las orquídeas de la selva baja no presentó diferencias significativas entre temporadas ni entre estratos (ANOVA anidada, $F_{2,18}=3, P=0.05$ para cada especie; Figura 2A-C). Únicamente, durante la temporada de sequía, se encontró que las hojas de Encyclia nematocaulon tuvieron un mayor CRA promedio en el estrato de $3.5 \mathrm{~m}(77 \pm 2 \%)$, que a $1.5 \mathrm{~m}(66 \pm 3 \% ; t=3.24$, g.l. $=4, P=0.05$; Figura 2A).

La acidificación nocturna $\left(\Delta \mathrm{H}^{+}\right)$de las hojas de las orquídeas epífitas fue significativamente diferente entre las temporadas de la selva baja (Kruskal-Wallis, $\mathrm{H}=26$, g.l. = $5, P=0.05$, para cada especie; Figura 2G-I). Por ejemplo, las hojas de Encyclia nematocaulon incrementaron notablemente la $\Delta \mathrm{H}^{+}$durante la temporada de nortes $(0.11 \pm 6 \mathrm{~mol}$ $\mathrm{H}^{+} \mathrm{m}^{-2}$ ) y decrecieron $90 \%$ en la temporada de sequía, sin diferencias significativas entre estratos (Figura 2G). El mismo patrón de la $\Delta \mathrm{H}^{+}$se observó en Cohniella yucatanensis y Laelia rubescens de la selva baja. Durante la sequía, el $\Psi_{\pi}$ de ambas especies fue más bajo a $3.5 \mathrm{~m}$ que a $1.5 \mathrm{~m}$, lo que coincidió con una tendencia en incremento de $\Delta \mathrm{H}^{+}$de las hojas de las orquídeas que crecieron a $3.5 \mathrm{~m}$.

La eficiencia cuántica máxima $\left(\mathrm{F}_{\mathrm{v}} / \mathrm{F}_{\mathrm{m}}\right)$ de las hojas de las orquídeas de la selva baja fue aproximadamente $0.8 \mathrm{du}-$ rante las temporadas de lluvias y nortes, pero decreció fuertemente en todas las especies durante la sequía $(\sim 0.46)$, lo que indica que presentaron fotoinhibición (Figura $2 \mathrm{~J}-\mathrm{L}$ ). La disminución de $\mathrm{F}_{\mathrm{v}} / \mathrm{F}_{\mathrm{m}}$ coincidió con el incremento de FFF y la disminución de $\Delta \mathrm{H}^{+}$en las especies de la selva baja. De igual manera, las hojas de las orquídeas de la selva baja 
Cuadro 1. Temperatura mínima y máxima y tasa de transporte de electrones máxima $\left(E_{T R}{ }_{\text {máx }}\right)$ de hojas de orquídeas de la selva baja caducifolia en Celestún, Yucatán, y de la selva mediana subcaducifolia en Kaxil-Kiuic, Yucatán, durante las temporadas de Iluvias, nortes y sequía. Los datos son medias $\pm E E\left(n=3\right.$ días para temperatura de la hoja y $n=3$ hojas para $\left.E T R_{\text {máx }}\right)$. Letras diferentes indican diferencias estadísticas entre temporadas para cada especie $(P=0.05)$.

\begin{tabular}{|c|c|c|c|c|c|c|c|}
\hline & \multicolumn{2}{|c|}{$\begin{array}{l}\text { Temperatura foliar } \\
\text { mínima }\left({ }^{\circ} \mathrm{C}\right)\end{array}$} & \multicolumn{2}{|c|}{$\begin{array}{c}\text { Temperatura foliar } \\
\text { máxima }\left({ }^{\circ} \mathrm{C}\right)\end{array}$} & \multicolumn{3}{|c|}{$\mathrm{ETR}_{\text {máx }}\left(\mu \mathrm{mol} \mathrm{m} \mathrm{m}^{-2} \mathrm{~s}^{-1}\right)$} \\
\hline & nortes & sequía & nortes & sequía & Iluvias & nortes & sequía \\
\hline \multicolumn{8}{|l|}{ Selva baja caducifolia } \\
\hline Encyclia nematocaulon & $22 \pm 0.5$ & $24 \pm 1$ & $29 \pm 2$ & $37 \pm 0.5$ & $18 \pm 1^{\mathrm{a}}$ & $11 \pm 1^{\mathrm{b}}$ & $5 \pm 0.3^{c}$ \\
\hline Cohniella yucatanensis & $22 \pm 0.5$ & $24 \pm 1$ & $29 \pm 2$ & $38 \pm 0.7$ & $10 \pm 1^{\mathrm{a}}$ & $10 \pm 1^{a}$ & $4 \pm 0.3^{b}$ \\
\hline Laelia rubescens & $22 \pm 0.5$ & $24 \pm 1$ & $29 \pm 2$ & $38 \pm 0.7$ & $14 \pm 0.5^{b}$ & $17 \pm 0.7^{a}$ & $10 \pm 0.5^{c}$ \\
\hline \multicolumn{8}{|c|}{ Selva mediana subcaducifolia } \\
\hline E. nematocaulon & $20 \pm 0.8$ & $23 \pm 0.7$ & $31 \pm 0.2$ & $38 \pm 0.7$ & $7 \pm 0.4^{\mathrm{b}}$ & $13 \pm 2^{\mathrm{a}}$ & $10 \pm 1^{\mathrm{a}}$ \\
\hline C. ascendens & $21 \pm 0.7$ & $21 \pm 0.1$ & $30 \pm 1$ & $34 \pm 0.2$ & $11 \pm 0.5^{b}$ & $20 \pm 4^{\mathrm{a}}$ & $16 \pm 1^{\mathrm{a}}$ \\
\hline Lophiaris oerstedii & $21 \pm 0.4$ & $23 \pm 0.9$ & $31 \pm 1$ & $37 \pm 0.5$ & $7 \pm 0.4^{\mathrm{b}}$ & $33 \pm 2^{\mathrm{a}}$ & $13 \pm 1^{\mathrm{b}}$ \\
\hline
\end{tabular}

mostraron los valores más bajos de ETR $_{\text {máx }}$ durante la sequía $\left(10 \mu \mathrm{mol} \mathrm{m}^{-2} \mathrm{~s}^{-1}\right.$; Cuadro 1$)$.

A diferencia de las orquídeas de la selva baja, las hojas de Encyclia nematocaulon y de Lophiaris oerstedii de la selva mediana mostraron cambios significativos en el CRA entre temporadas (ANOVA anidada, $F_{2,18}=80, P=0.05$ para ambas especies; Figura 3A, C); el CRA de L. oerstedii disminuyó $40 \%$ durante la temporada de sequía en comparación

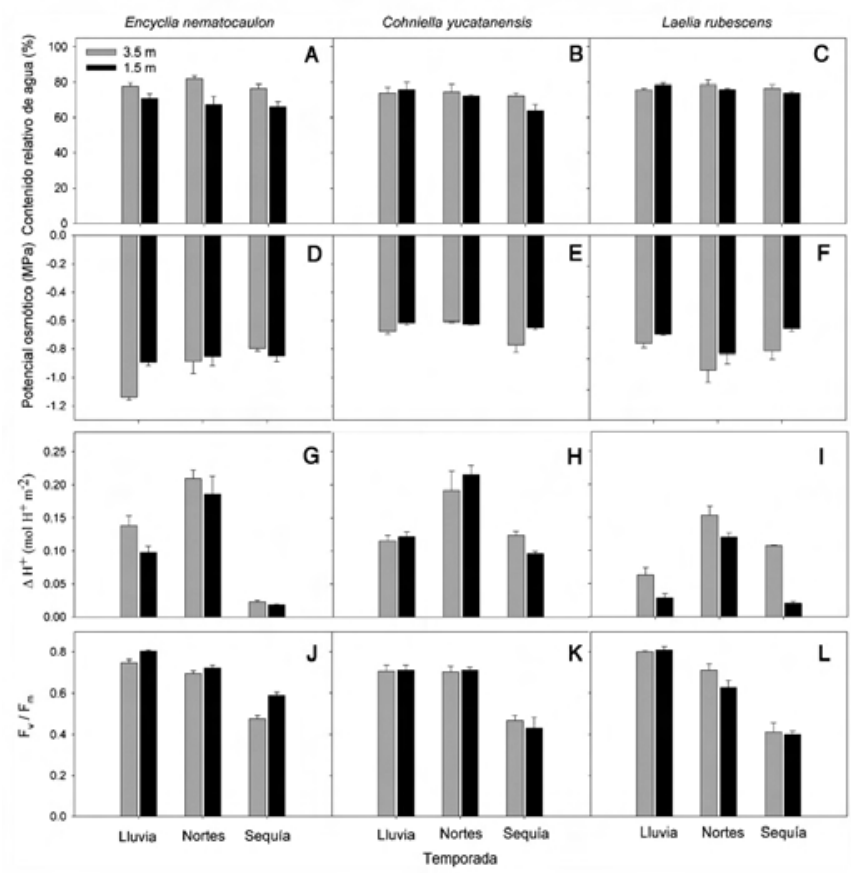

Figura 2. Cambios estacionales del contenido relativo de agua $(\%)$, potencial osmótico $(\mathrm{MPa})$, acidificación nocturna $\left(\Delta \mathrm{H}^{+}\right)$y eficiencia cuántica máxima $\left(\mathrm{F}_{\mathrm{v}} / \mathrm{F}_{\mathrm{m}}\right)$, de orquídeas epífitas en dos alturas en el hospedero ( 3.5 y $1.5 \mathrm{~m}$ ) de la selva baja caducifolia de Celestún. Los datos son medias $\pm \mathrm{EE}, \mathrm{n}=5$. con la temporada de lluvias (Figura 3C), sin diferencias significativas entre estratos en ambas temporadas $(P=0.05)$. La especie que crece en ambos sitios, E. nematocaulon, presentó en promedio $70 \pm 1 \%$ de CRA en la selva baja y $90 \pm$ $3 \%$ de CRA en la selva mediana, durante la temporada de sequía $(t=6.22$, g.1. $=10, P=0.05$; Figuras $2 \mathrm{~A}, 3 \mathrm{~A})$.

En la selva mediana, solo las hojas de Encyclia nematocaulon mostraron cambios estacionales en la $\Delta \mathrm{H}^{+}$(ANOVA

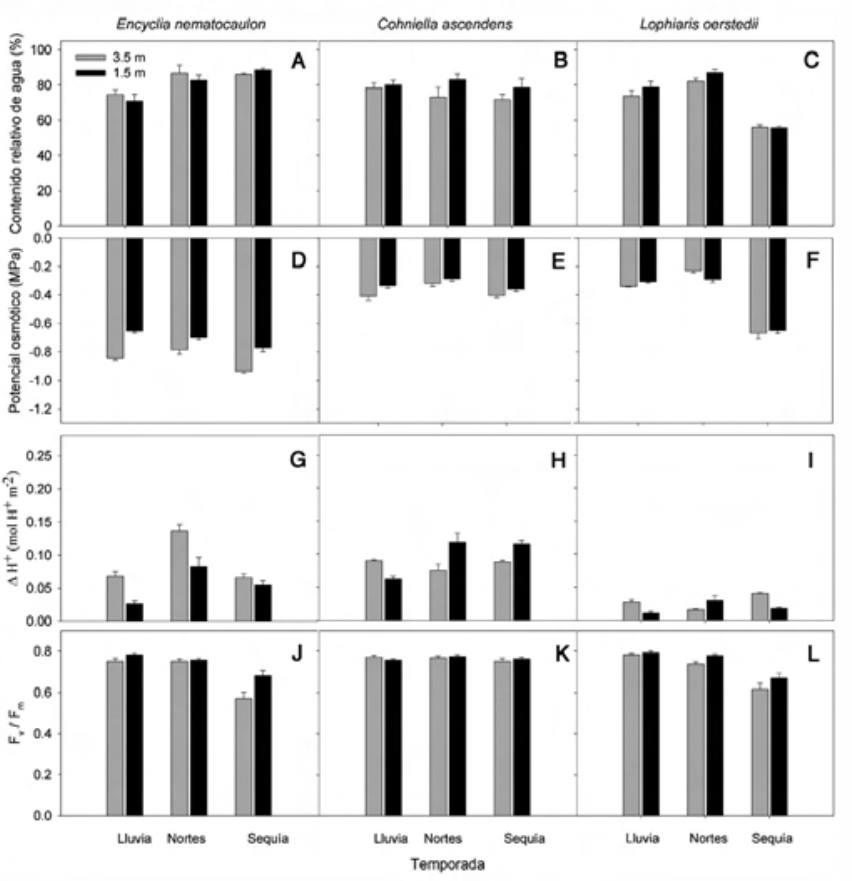

Figura 3. Cambios estacionales del contenido relativo de agua $(\%)$, potencial osmótico $(\mathrm{MPa})$, acidificación nocturna $\left(\Delta \mathrm{H}^{+}\right)$y eficiencia cuántica máxima $\left(\mathrm{F}_{\mathrm{v}} / \mathrm{F}_{\mathrm{m}}\right)$ de orquídeas epífitas en dos alturas en el hospedero ( 3.5 y $1.5 \mathrm{~m}$ ) de la selva mediana subcaducifolia de Kaxil-Kiuic. Los datos son medias \pm EE, $n=5$. 
Cuadro 2. Volumen de pseudobulbos, densidad de estomas, área foliar específica y forma de la hoja de orquídeas epífitas de la selva baja caducifolia de Celestún, Yucatán, y de la selva mediana subcaducifolia de Kaxil-Kiuic, Yucatán. Los datos son medias \pm EE ( $n=10$ para volumen de pseudobulbos, $n=4$ para densidad de estomas y $n=21-34$ para área foliar específica). Letras diferentes indican diferencias estadísticas entre las especies de cada selva $(P=0.05)$.

\begin{tabular}{|c|c|c|c|c|c|}
\hline & \multirow{2}{*}{$\begin{array}{l}\text { Volumen de } \\
\text { pseudobulbos } \\
\left(\mathrm{cm}^{3}\right)\end{array}$} & \multicolumn{2}{|c|}{$\begin{array}{l}\text { Densidad de } \\
\text { estomas }\left(\mathrm{mm}^{2}\right)\end{array}$} & \multirow{2}{*}{$\begin{array}{l}\text { Área foliar } \\
\text { específica } \\
\left(\mathrm{cm}^{2} \mathrm{~g}^{-1}\right)\end{array}$} & \multirow[t]{2}{*}{$\begin{array}{c}\text { Forma de } \\
\text { la hoja }\end{array}$} \\
\hline & & adaxial & abaxial & & \\
\hline \multicolumn{6}{|l|}{ Selva baja caducifolia } \\
\hline Encyclia nematocaulon & $0.44 \pm 0.06$ & 0 & $60 \pm 2^{\mathrm{a}}$ & $48.9 \pm 1.6^{a}$ & lineal \\
\hline Cohniella yucatanensis & $0.24 \pm 0.04$ & 0 & $21 \pm 2^{\mathrm{c}}$ & $46.5 \pm 3.1^{\mathrm{a}}$ & cilíndrica \\
\hline Laelia rubescens & $5.40 \pm 0.66$ & 0 & $50 \pm 3^{b}$ & $59.6 \pm 3.6^{\mathrm{b}}$ & ovalada \\
\hline \multicolumn{6}{|c|}{ Selva mediana subcaducifolia } \\
\hline E. nematocaulon & $0.45 \pm 0.04$ & 0 & $61 \pm 4^{\mathrm{a}}$ & $87.1 \pm 4.3^{\mathrm{a}}$ & lineal \\
\hline C. ascendens & $0.17 \pm 0.03$ & 0 & $27 \pm 1^{\mathrm{c}}$ & $66.1 \pm 4.1^{\mathrm{b}}$ & cilíndrica \\
\hline Lophiaris oerstedii & $0.16 \pm 0.03$ & $18 \pm 1$ & $23 \pm 1^{\mathrm{b}}$ & $43.1 \pm 1.4^{\mathrm{b}}$ & ovalada \\
\hline
\end{tabular}

anidada, $F_{2}, 18=26, P=0.05$; Figura $\left.3 \mathrm{G}\right)$. Durante la sequía, la $\Delta \mathrm{H}^{+}$disminuyó 50 y $30 \%$ a 3.5 y $1.5 \mathrm{~m}$ de altura en el hospedero, respectivamente, en comparación con la temporada de nortes (Figura 3G). Por otro lado, las hojas de Lophiaris oerstedii mostraron la $\Delta \mathrm{H}^{+}$más baja $\left(0.04 \mathrm{~mol} \mathrm{H}^{+}\right.$ $\mathrm{m}^{-2}$ ) durante las tres temporadas. En la temporada de sequía, en E. nematocaulon de la selva mediana, la $\Delta \mathrm{H}^{+}$y la $\mathrm{ETR}_{\text {máx }}$ de sus hojas se incrementaron significativamente en comparación con las plantas de la misma especie en la selva baja (Kruskal-Wallis, $\mathrm{H}=14.2$, g.l. $=1, P=0.05$, para ambas variables).

$\mathrm{Al}$ igual que las especies de la selva baja, las hojas de Encyclia nematocaulon y Lophiaris oerstedii disminuyeron $\mathrm{F}_{\mathrm{v}} / \mathrm{F}_{\mathrm{m}}$ durante la temporada de sequía $(\sim 0.63)$ sin diferencias significativas entre estratos $(P=0.05$; Figura $3 \mathrm{~J}, 1)$. Cabe destacar que Cohniella ascendens, de la selva mediana, fue la única especie que no presentó variación estacional en las variables CRA, $\Delta \mathrm{H}^{+}, \Psi_{\pi}$ y $\mathrm{F}_{\mathrm{v}} / \mathrm{F}_{\mathrm{m}}$.

Volumen de pseudobulbos, densidad estomática, y área foliar específica. En la selva baja, Laelia rubescens tuvo el mayor volumen de los pseudobulbos, el cual fue diez veces mayor que para Encyclia nematocaulon y Cohniella yucatanensis. Mientras que en la selva mediana, el volumen de los pseudobulbos de E. nematocaulon fue dos veces mayor que los de C. ascendens y Lophiaris oerstedii (Cuadro 2).

En ambas selvas, las hojas de Encyclia nematocaulon tuvieron la mayor densidad estomática de todas las especies $\left(\sim 60 \mathrm{~mm}^{2}\right)$, sin diferencias significativas entre selvas $(t=$ 0.23 , g.1. $=6, P=0.05$; Cuadro 2). En la selva baja, E. nematocaulon y Cohniella yucatanensis presentaron hojas con un área foliar específica (AFE) menor que las de L. rubescens (Kruskal-Wallis, $\mathrm{H}=6.06$, g.l. $=1, P=0.05$; Cuadro 2), mientras que en la selva mediana, el AFE de E. nematocaulon fue mayor que el de las otras dos especies y dos veces mayor que el de Lophiaris oerstedii (Kruskal-Wallis, H =
58.5, g.1. $=1, P=0.05$; Cuadro 2). La especie que crece en ambas selvas, E. nematocaulon, tuvo hojas con una AFE $40 \%$ mayor en la selva mediana que en la selva baja.

Microscopía electrónica de barrido. Se observaron estomas en la superficie abaxial de la hojas de todas las orquídeas (Figura 4), solo Lophiaris oerstedii presentó estomas en ambas superficies. Los estomas de Encyclia nematocaulon fueron los más pequeños en ambas selvas $\left(558 \pm 16 \mu \mathrm{m}^{2}\right.$; Figura 4B, H) y los de Laelia rubescens fueron los más grandes $\left(1,865 \pm 61 \mu \mathrm{m}^{2}\right.$; Figura $\left.4 \mathrm{~F}\right)$. La superficie de la epidermis de Cohniella yucatanensis y C. ascendens presentó ondulaciones irregulares con papilas (Figura 4C, E, I); en la primera especie los estomas aparecen hundidos en la epidermis (Figura 4D).

\section{Discusión}

La mayoría de las orquídeas estudiadas mostraron variación fisiológica ante la estacionalidad, más que entre estratos en ambas selvas. Sorprendentemente y contrario a la hipótesis planteada, el contenido relativo de agua (CRA) foliar de las orquídeas de la selva baja fue constante durante las temporadas de lluvias, nortes y sequía, a pesar de que las orquídeas recibieron dos veces más luz durante la sequía que en la temporada de lluvias. El control estomático y la fotosíntesis CAM fueron efectivos para evitar la pérdida de agua por transpiración (Lambers et al., 1998), a pesar del aumento en la demanda evaporativa durante esa época. Se ha demostrado que en sitios con una demanda evaporativa alta disminuye la permeabilidad de la cutícula epidérmica, lo cual reduce la transpiración (Helbsing et al., 2000). Si se considera que algunas orquídeas epífitas pierden $\sim 200 \mu \mathrm{mol}$ $\mathrm{H}_{2} \mathrm{O} \mathrm{m}^{-2} \mathrm{~s}^{-1}$ por transpiración (Goh y Kluge, 1989) y que la deposición de rocío en las orquídeas de la selva baja es de $\sim 0.03 \mathrm{~g} \mathrm{H}_{2} \mathrm{O} \mathrm{m}^{-2} \mathrm{~s}^{-1}$ durante la temporada de sequía (datos 

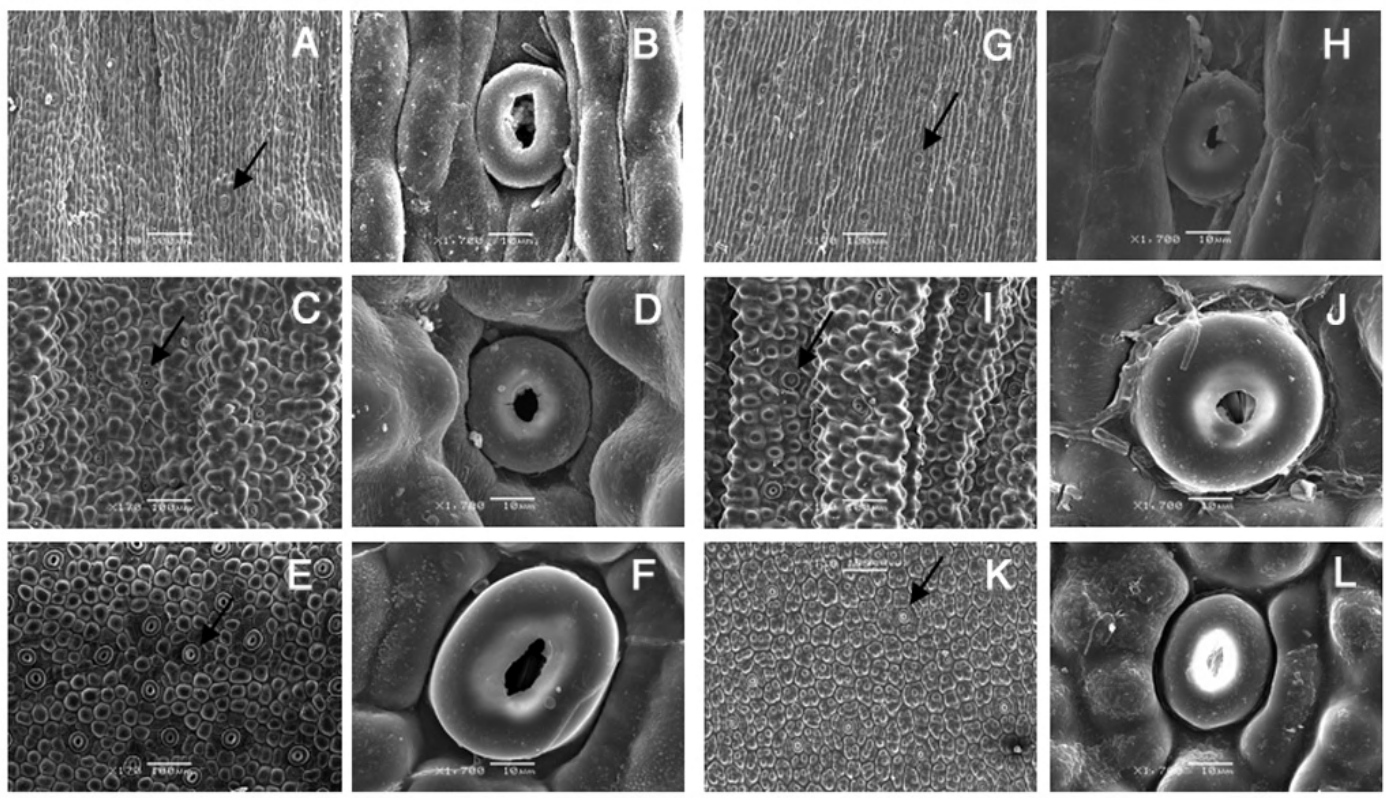

Figura 4. Microfotografías del microscopio electrónico de barrido que muestran las secciones de epidermis y los estomas de orquídeas epífitas de la selva baja caducifolia: Encyclia nematocaulon (A-B), Cohniella yucatanensis (C-D) y Laelia rubescens (E-F) y de la selva mediana subcaducifolia: E. nematocaulon $(\mathrm{G}-\mathrm{H})$, C. ascendens $(\mathrm{I}-\mathrm{J})$ y Lophiaris oerstedii $(\mathrm{K}-\mathrm{L})$. La línea blanca representa la escala. La flecha indica la ubicación de los estomas.

no publicados), se puede inferir que las orquídeas podrían perder poca agua durante esta temporada sin variar su CRA. Los valores constantes del CRA pueden ser explicados por la suculencia de sus pseudobulbos, los cuales pueden llegar a almacenar hasta un $95 \%$ del agua de toda la planta (Benzing, 1990; Zimmerman, 1990; Zotz y Hietz, 2001). En la selva baja, Laelia rubescens tuvo el mayor volumen de pseudobulbo, el cual fue diez veces mayor en comparación con aquéllos de Encyclia nematocaulon y Cohniella yиcatanensis (Cuadro 2). El pseudobulbo de L. rubescens le permitió transportar agua hacia sus hojas anchas para mantener el potencial hídrico alto, realizar la fotosíntesis y evitar el sobrecalentamiento foliar (Schäfer y Lüttge, 1986; Zimmerman, 1990).

Por otro lado, las orquídeas con menor volumen de pseudobulbos (Cohniella yucatanensis, C. ascendens y Lophiaris oerstedii) tuvieron hojas más suculentas que las otras especies. La suculencia de las hojas de muchas epífitas, particularmente las orquídeas, se debe a una hipodermis que funciona como un almacén de agua (Oliveira y Sajo, 1999), y en algunas especies puede ocupar hasta el $80 \%$ del volumen de la hoja (Pridgeon, 1986). Otra característica común en los tejidos foliares de las orquídeas es la presencia de idioblastos, estructuras engrosadas que acumulan agua y evitan el colapso de los tejidos ante el déficit hídrico (Sandoval-Zapotitla y Terrazas, 2001; Evert, 2006).

Las orquídeas de la selva baja y Encyclia nematocaulon, de la selva mediana, exhibieron un notable incremento de acidificación nocturna $\left(\Delta \mathrm{H}^{+}\right)$en la temporada de nortes. En esta temporada, el DPV mínimo, el FFF y la temperatura mínima foliar fueron más bajos en comparación con la temporada de sequía; además, la formación de rocío o neblina puede ocurrir en esta temporada (Andrade, 2003; ReyesGarcía et al., 2012), que son condiciones que favorecen la apertura estomática e incrementan la captura de $\mathrm{CO}_{2}$. Asimismo, las orquídeas mostraron valores de $\mathrm{F}_{\mathrm{v}} / \mathrm{F}_{\mathrm{m}}$ cercanos al óptimo téorico (0.8-0.83; Björkman y Demmig, 1987), indicativo de que las plantas no estuvieron bajo algún tipo de estrés ambiental durante el periodo de nortes (Horton $e t$ al., 1996; Demmig-Adams et al., 2012). Valores similares de $\Delta \mathrm{H}^{+}$, registrados en la temporada de nortes $(0.15-0.56$ mol $\mathrm{H}^{+} \mathrm{m}^{-2}$ ), han sido reportados para otras orquídeas epífitas en diferentes ambientes, en condiciones de campo y de laboratorio (McWilliams, 1970; Goh et al., 1977; Winter et al., 1983; Sinclair, 1984; Winter et al., 1986).

Por otro lado y de acuerdo a nuestra predicción, la mayoría de las especies disminuyeron la $\Delta \mathrm{H}^{+}$durante la temporada de sequía, cuando las condiciones ambientales como el FFF, el DPV y la temperatura foliar (Cuadro 1) aumentaron; lo que fue consistente con la disminución de la eficiencia del transporte de electrones $\left(\right.$ ETR $\left._{\text {máx }}\right)$ y la eficiencia cuántica máxima $\left(\mathrm{F}_{\mathrm{v}} / \mathrm{F}_{\mathrm{m}}\right)$. Se ha reportado que valores de $\mathrm{F}_{\mathrm{v}} / \mathrm{F}_{\mathrm{m}}$ entre 0.4 y 0.7 indican fotoinhibición moderada, un proceso que reduce la captura de $\mathrm{CO}_{2}$ debido al daño de los centros de reacción del fotosistema II (Björkman y Demmig, 1987; Lambers et al., 1998; Adams et al., 2008). Posiblemente las hojas lineares de Encyclia nematocaulon tengan mayor control estomático que las hojas anchas de Laelia rubescens y 
cilíndricas de Cohniella yucatanensis, respectivamente, ya que disminuyeron fuertemente su $\Delta \mathrm{H}^{+}(90 \%)$ como una estrategia para mantener el balance hídrico (Griffiths et al., 1989; Benzing, 1990; Andrade-Souza et al., 2009; Nobel, 2009). Al considerar una estequiometria de $2 \mathrm{~mol} \mathrm{H}^{+}: 1 \mathrm{~mol}$ $\mathrm{CO}_{2}$ (Winter, 1985; Zotz y Tyree, 1996), el promedio estacional de $\Delta \mathrm{H}^{+}$de E. nematocaulon durante la sequía ( 0.023 $\mathrm{mol} \mathrm{H} \mathrm{H}^{+} \mathrm{m}^{-2}$ ) debería corresponder a una tasa de fijación de $\mathrm{CO}_{2}$ de $\sim 3 \mu \mathrm{mol} \mathrm{m}^{-2} \mathrm{~s}^{-1}$ en un periodo de $1 \mathrm{~h}$, este valor es similar al que presenta la orquídea epífita Dimerandra emarginata bajo sequía (Zotz y Tyree, 1996), pero este valor es mayor al promedio de algunas orquídeas CAM $\left(1.7 \mu \mathrm{mol} \mathrm{m} \mathrm{m}^{-2} \mathrm{~s}^{-1}\right.$; Stuntz y Zotz, 2001). La asimilación de $\mathrm{CO}_{2}$ en E. nematocaulon pudo solo ocurrir en pre-alba (Andrade y Nobel, 1997; Andrade et al., 2007; Nobel, 2009); o bien, fue el resultado del reciclaje interno de $\mathrm{CO}_{2}$ (Griffiths et al., 1989). Se sugiere realizar mediciones de la asimilación de $\mathrm{CO}_{2}$ para conocer los ajustes fotosintéticos de estas especies.

A pesar de las diferencias morfológicas foliares, las orquídeas de la selva baja mostraron una variación fisiológica similar ante la estacionalidad. Sin embargo, las orquídeas de la selva mediana mostraron distintas estrategias fisiológicas. Por ejemplo, Cohniella ascendens, la cual crece en zonas sombreadas (Cetzal-Ix y Carnevali, 2010), no presentó variaciones significativas del $\mathrm{CRA}, \Delta \mathrm{H}^{+}, \Psi_{\pi}$ y $\mathrm{F}_{\mathrm{v}} / \mathrm{F}_{\mathrm{m}}$ entre temporadas ni entre estratos. Se sugiere que la posición semi-péndula de sus hojas (Cetzal-Ix et al., 2013) y su epidermis irregular (Figura 4I) hacen que evite la luz directa $\mathrm{y}$, como consecuencia, la fotoinhibición; además, su baja densidad estomática podría contribuir a mantener el balance hídrico. Por su parte, Lophiaris oerstedii, que habita en la selva mediana, fue la única especie que disminuyó $40 \%$ su CRA en los dos estratos en la temporada de sequía, posiblemente se deba a la distribución de sus estomas en ambas superficies de la hoja (Sandoval-Zapotitla y Terrazas, 2001), y tal vez a un menor control estomático y una mayor área de exposición, lo que le confiere una mayor susceptibilidad a la pérdida de agua durante la sequía. Se sugiere que C. ascendens y L. oerstedii podrían ser más sensibles a los cambios ambientales; un incremento sustancial de luz, como el que ocurre en la selva baja, podría afectar negativamente su estado hídrico y fotosintético; posiblemente la selva mediana representa el límite más seco de la distribución de estas especies.

La única especie que crece en las dos selvas, Encyclia nematocaulon, desplegó una importante variación de respuestas morfo-fisiológicas, lo que demostró su plasticidad para ocupar distintos estratos en el hospedero, incluso en lo alto del dosel en ambas selvas. Esto se refleja, por ejemplo, en el área foliar específica (AFE) de esta especie, que fue mayor en la selva mediana en comparación con la selva baja. Esto coincide con el hecho de que las plantas que crecen en sitios sombreados, generalmente presentan hojas más del- gadas y mayor AFE, como una adaptación fotosintética para capturar fotones (Poorter y De Jong, 1999). En cambio, las plantas que crecen en sitios soleados, generalmente tienen hojas gruesas con baja AFE (Björkman, 1981; Marcelis et al., 1998), lo que disminuye la exposición del área foliar al FFF y reducen la fotosíntesis y el crecimiento; lo anterior, además de las hojas pequeñas, representan estrategias para disminuir la transpiración e incrementar el uso eficiente del agua (Lambers et al., 1998; Craufurd et al., 1999).

\section{Conclusiones}

Las orquídeas epífitas de este estudio presentaron una mayor variación fisiológica entre temporadas que entre estratos, a pesar que el ambiente lumínico fue también diferente a nivel de estratos en los dos sitios de estudio. La regulación del balance hídrico y el posible control estomático de las orquídeas durante la sequía podrían representar una estrategia de tolerancia a las condiciones desecantes, lo que explica su distribución en la selva baja (Andrade et al., 2007). Al mismo tiempo, Cohniella ascendens y Lophiaris oerstedii, de la selva mediana, podrían ser más susceptibles al cambio climático debido a su estrecha variación fisiológica; se sugiere que la exposición de estas especies a diferentes condiciones controladas de luz y agua podría revelarnos su sensibilidad fisiológica. Finalmente, Encyclia nematocaulon mostró una gran plasticidad fisiológica ante la estacionalidad que ocurre en las dos selvas, lo cual podría relacionarse con su abundancia en dichas selvas.

\section{Agradecimientos}

Agradecemos a Roberth Us y Luis Simá por su ayuda en campo y por la obtención de los datos micrometereológicos. A Lilia Can por su ayuda con las microfotografías del MEB. También agradecemos al dueño del rancho en Celestún y al administrador de la Reserva Biocultural KaxilKiuic Helen Moyers por las facilidades para realizar este estudio. Especialmente agradecemos a Fernando Arellano, Diana Cisneros, María Cabañas y a dos revisores anónimos por comentarios a una previa versión del manuscrito. E. de la Rosa-Manzano recibió una beca por parte del Consejo Nacional de Ciencia y Tecnología, México (número 204452).

\section{Literatura citada}

Adams W.W. III, Zarter C.R., Mueh K.E., Amiard V.S.E. y Demmig-Adams B. 2008. Energy dissipation and photoinhibition: a continuum of photoprotection. En Demmig-Adams B., Adams W.W.III y Matto A. Eds. Photoprotection, Photoinhibition, Gene Regulation and Environment, pp. 49-64, Springer, Berlín.

Andrade J.L. 2003. Dew deposition on epiphytic bromeliad leaves: an important event in a Mexican tropical dry deciduous forest. Journal of Tropical Ecology 19:479-488. 
Andrade J.L. y Nobel P.S. 1997. Microhabitats and water relation of epiphytic cacti and ferns in a lowland Neotropical forest. Biotropica 29:261-270.

Andrade J.L., de la Barrera E., Reyes-García C., Ricalde M.F., Vargas-Soto G. y Cervera J.C. 2007. El metabolismo ácido de las crasuláceas: diversidad, fisiología ambiental y productividad. Boletín de la Sociedad Botánica de México 81:37-50.

Andrade J.L., Rengifo E., Ricalde M.F., Simá J.L., Cervera J.C. y Vargas-Soto G. 2006. Microambientes de luz, crecimiento y fotosíntesis de la pitahaya (Hylocereus undatus) en un agrosistema de Yucatán, México. Agrociencia 40:687-697.

Andrade-Souza V., Almeida A.A.F., Corrêa R.X., Costa M.A., Mielke M.S. y Gomes F.P. 2009. Leaf carbon assimilation and molecular phylogeny in Cattleya species (Orchidaceae). Genetics and Molecular Research 8:976-989.

Benzing D.H. 1990. Vascular Epiphytes. Cambridge University Press, Cambridge.

Björkman O. 1981. Responses to different quantum flux densities. En: Lange O.L., Nobel P.S., Osmond C.B. y Ziegler H. Eds. Physiological Plant Ecology I. Responses to the Physical Environment, Encyclopedia of Plant Physiology New Series Vol. 12A, pp. 57-107, Springer-Verlag, Berlín.

Björkman O. y Demmig B. 1987. Photon yield of $\mathrm{O}_{2}$ evolution and chlorophyll fluorescence characteristics at $77 \mathrm{~K}$ among vascular plants of diverse origins. Planta 170:489-504.

Carnevali F.C.G., Tapia-Muñoz J.L., Jiménez-Machorro R., Sánchez-Saldaña L., Ibarra-González L., Ramírez I.M. y Gómez M.P. 2001. Notes on the flora of the Yucatan Peninsula II: a synopsis of the orchid flora of the Mexican Yucatan Peninsula and a tentative checklist of the Orchidaceae of the Yucatan Peninsula Biotic Province. Harvard Papers in Botany 5:383-466.

Cetzal-Ix W. y Carnevali F.C.G. 2010. A revision of Cohniella Pfitzer (Orchidaceae) in Mexico. Journal of the Torrey Botanical Society 137:180-213.

Cetzal-Ix W., Carnevali G., Noguera-Savelli E. y Jauregui D. 2013. Morphological and anatomical characterization of a new natural hybrid between Cohniella ascendens and C. brachyphylla (Oncidiinae; Orchidaceae). Phytotaxa 144:45-55.

Craufurd P.Q., Wheeler T.R., Ellis R.H., Summerfield R.J. y Williams J.H. 1999. Effect of temperature and water deficit on water-use efficiency, carbon isotope discrimination, and specific leaf area in peanut. Crop Science 39:136-142.

Demmig-Adams B. y Adams W.W.III. 1992. Photoprotection and other responses of plants to high light stress. Annual Review of Plant Physiology and Plant Molecular Biology 43:599-626.

Demmig-Adams B., Cohu C.M., Muller O. y Adams W.W.III. 2012. Modulation of photosynthetic energy conversion efficiency in nature: from seconds to seasons. Photosynthesis Research 113:75-88.

Dupuy J.M., Hernández-Stefanoni J.L., Hernádez-Juárez R.A., Tetetla-Rangel E., López-Martínez J.O., Leyequién-Abarca E., Tun-Dzul F.J. y May-Pat F. 2012. Patterns and correlates of tropical dry forest structure and composition in a highly replicated chronosequences in Yucatan, Mexico. Biotropica 44:151-162.

Evert R.F. 2006. Esau's Plant Anatomy. Meristems, Cells, and Tissues of the Plant Body: their Structure, Function, and Development. John Wiley \& Sons, Nueva Jersey.

Gentry A.H. y Dodson C. 1987. Contribution of nontrees to species richness of a tropical rain forest. Biotropica 19:149-156.
Goh C.J. y Kluge M. 1989. Gas Exchange and water relations in epiphytic orchids. En: Lüttge U. Ed. Vascular Plants as Epiphytes. Evolution and Ecophysiology, pp 139-166, SpringerVerlag, Berlín.

Goh C.J., Avadhani P.N., Loh C.S., Hanegraaf C. y Arditi J. 1977. Diurnal stomatal and acidity rhythms in orchid leaves. New Phytologist 78:365-372.

González-Salvatierra C., Andrade J.L., Orellana R., Peña-Rodríguez L.M. y Reyes-García C. 2013. Microambiente lumínico y morfología y fisiología foliar de Bromelia karatas (Bromeliaceae) en una selva baja caducifolia de Yucatán, México. Botanical Sciences 91:75-84.

Graham E.A. y Andrade J.L. 2004. Drought tolerance associated with vertical stratification of two co-occurring epiphytic bromeliads in a tropical dry forest. American Journal of Botany 91:699-706.

Griffiths H. y Smith J.A.C. 1983. Photosynthetic pathways in the Bromeliaceae of Trinidad: relations between life-forms, habitat preference and the occurrence of CAM. Oecologia 60:176184.

Griffiths H., Ong B.L., Avadhani P.N. y Goh C.J. 1989. Recycling of respiratory $\mathrm{CO}_{2}$ during crasssulacean acid metabolism: alleviation of photoinhibition in Pyrrosia piloselloides. Planta 179:115-122.

Helbsing S., Riederer M. y Zotz G. 2000. Cuticles of vascular epiphytes: efficient barriers for water loss after stomatal closure? Annals of Botany 86:765-769.

Horton P., Ruban A.V. y Walters R.G. 1996. Regulation of light harvesting in green plants. Annual Review of Plant Physiology and Plant Molecular Biology 47:655-684.

Jones H.G. 1992. Plant and Microclimate: A Quantitative Approach to Environmental Plant Physiology. Cambridge University Press, Cambridge.

Lambers H., Chapin F.S.III y Pons T.L. 1998. Plant Physiological Ecology. Springer-Verlag, Nueva York.

Marcelis L.F.M., Heuvelink E. y Goudriaan J. 1998. Modelling biomass production and yield of horticultural crops: a review. Scientia Horticulturae 74:83-111.

Maxwell K. y Johnson G.N. 2000. Chlorophyll fluorescence-a practical guide. Journal of Experimental of Botany 51:659668.

McWilliams E.L. 1970. Comparative rates of dark $\mathrm{CO}_{2}$ uptake and acidification in the Bromeliaceae, Orchidaceae, and Euphorbiaceae. Botanical Gazette 131:285-290.

Nobel P.S. 2009. Physicochemical and Environmental Plant Physiology. $4^{\mathrm{a}}$ ed. Academic Press, Oxford.

Oliveira V.C. y Sajo M.G. 1999. Leaf anatomy of epiphyte species of Orchidaceae. Brazilian Journal of Botany 22:365-374.

Orellana R. 1999. Evaluación climática. En: García S.A., Chico P.L.P. y Orellana L.R.. Eds. Atlas de Procesos Territoriales de Yucatán, pp. 163-182, Facultad de Arquitectura, Universidad Autónoma de Yucatán, Mérida.

Poorter H. y De Jong R. 1999. A comparison of specific leaf area, chemical composition and leaf construction costs of field plants from 15 habitats differing in productivity. New Phytologist 143:163-176.

Powles S.B. 1984. Photoinhibition of photosynthesis induced by visible light. Annual Review of Plant Physiology 35:15-44.

Pridgeon A.M. 1986. Anatomical adaptations in Orchidaceae. Lindleyana 1:90-101. 
Rascher U., Liebig M. y Lüttge U. 2000. Evaluation of instant light-response curves of chlorophyll fluorescence parameters obtained with a portable chlorophyll fluorometer on site in the field. Plant, Cell and Environment 23:1397-1405.

Reyes-García C., Mejia-Chang M. y Griffiths H. 2012. High but not dry: diverse epiphytic bromeliad adaptations to exposure within a seasonally dry tropical forest community. New Phytologist 193:745-754.

Sandoval-Zapotitla E. y Terrazas T. 2001. Leaf anatomy of 16 taxa of the Trichocentrum clade (Orchidaceae: Oncidiinae). Lindleyana 16:81-93.

Schäfer C. y Lüttge U. 1986. Effects of water stress on gas exchange and water relations of a succulent epiphyte, Kalanchö̈ uniflora. Oecologia 71:127-132.

Sinclair R. 1984. Water relations of tropical epiphytes: III. Evidence for crassulacean acid metabolism. Journal of Experimental of Botany 35:1-7.

Stuntz S. y Zotz G. 2001. Photosynthesis in vascular epiphytes: a survey of 27 species of diverse taxonomic origin. Flora 196:132-141.

Trejo I. y Dirzo R. 2000. Deforestation of seasonally dry tropical forest: a national and local analysis in Mexico. Biological Conservation 94:133-142.

Recibido: 6 de octubre de 2013

Aceptado: 3 de enero de 2014
Winter K. 1985. Crassulacean acid metabolism. En: Barber J. y Baker N.R. Eds. Photosynthetic Mechanisms and the Environment Topics in Photosynthesis, pp 329-387, Elsevier, Amsterdam.

Winter K., Osmond C.B. y Hubick K.T. 1986. Crassulacean acid metabolism in the shade. Studies on an epiphytic fern, Pyrrosia longifolia, and other rainforest species from Australia. Oecologia 68:224-230.

Winter K., Wallace B.J., Stocker G.C. y Roksandic Z. 1983. Crassulacean acid metabolism in Australian vascular epiphytes and some related species. Oecologia 57:129-141.

Zimmerman J.K. 1990. Role of pseudobulbs in growth and flowering of Catasetum viridiflavum (Orchidaceae). American Journal of Botany 77:533-542.

Zotz G. y Andrade J.L. 1998. Water relations of two co-ocurring epiphytic bromeliads. Journal of Plant Physiology 152:545549, 552-554.

Zotz G. y Hietz P. 2001. The physiological ecology of vascular epiphytes: current knowledge, open questions. Journal of Experimental Botany 52:2067-2078.

Zotz G. y Tyree M.T. 1996. Water stress in the epiphytic orchid, Dimerandra emarginata (G. Meyer) Hoehne. Oecologia 107:151-159. 